\title{
REVIEW
}

\section{South African food allergy consensus document 2014}

\author{
M E Levin, C L Gray, E Goddard, S Karabus, M Kriel, A C Lang, A I Manjra, S M Risenga, A J Terblanche, D A van der Spuy, \\ for the South African Food Allergy Working Group (SAFAWG)
}

All authors' degrees, affiliations and conflict of interest statements can be found online at http://dx.doi.org/10.7196/SAMJ.9201

Corresponding author: M Levin (michael.levin@uct.ac.za)

The prevalence of food allergy is increasing worldwide and is an important cause of anaphylaxis. There are no local South African food allergy guidelines. This document was devised by the Allergy Society of South Africa (ALLSA), the South African Gastroenterology Society (SAGES) and the Association for Dietetics in South Africa (ADSA).

Subjects may have reactions to more than one food, and different types and severity of reactions to different foods may coexist in one individual. A detailed history directed at identifying the type and severity of possible reactions is essential for every food allergen under consideration. Skin-prick tests and specific immunoglobulin E (IgE) (ImmunoCAP) tests prove IgE sensitisation rather than clinical reactivity. The magnitude of sensitisation combined with the history may be sufficient to ascribe causality, but where this is not possible an incremental oral food challenge may be required to assess tolerance or clinical allergy. For milder non-IgE-mediated conditions a diagnostic elimination diet may be followed with food re-introduction at home to assess causality.

The primary therapy for food allergy is strict avoidance of the offending food/s, taking into account nutritional status and provision of alternative sources of nutrients. Acute management of severe reactions requires prompt intramuscular administration of adrenaline $0.01 \mathrm{mg} / \mathrm{kg}$ and basic resuscitation. Adjunctive therapy includes antihistamines, bronchodilators and corticosteroids. Subjects with food allergy require risk assessment and those at increased risk for future severe reactions require the implementation of risk-reduction strategies, including education of the patient, families and all caregivers (including teachers), the provision of a written emergency action plan, a MedicAlert necklace or bracelet and injectable adrenaline (preferably via auto-injector) where necessary.

S Afr Med J 2015;105(1):62-65. DOI:10.7196/SAMJ.9098

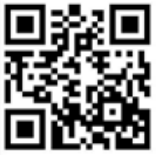

Up to $34 \%$ of individuals or parents think that they or a family member has a food allergy and $22 \%$ avoid particular foods because of the mere possibility that the food may contain an allergen, when in fact only between $1 \%$ and $6 \%$ test positive on full evaluation. ${ }^{[1]}$

A working group was constituted of medical professionals with interest and expertise in food allergy in South Africa (SA) with representation from the Allergy Society of South Africa (ALLSA), the South African Gastroenterology Society (SAGES) and the Association for Dietetics in South Africa (ADSA). The structure and content of the document was networked, individuals were allocated sections to write and a draft document was compiled. A meeting was then held to discuss the draft document, resolve controversial issues and achieve a consensus document. The document is endorsed by ALLSA, SAGES and ADSA.

\section{Definitions}

Adverse reactions to food can be divided into psychological reactions (food aversion), organic reactions (e.g. peptic ulcer disease), anatomical reactions (e.g. strictures), toxic reactions (e.g. food poisoning) and nontoxic reactions (Fig. 1). ${ }^{[2]}$

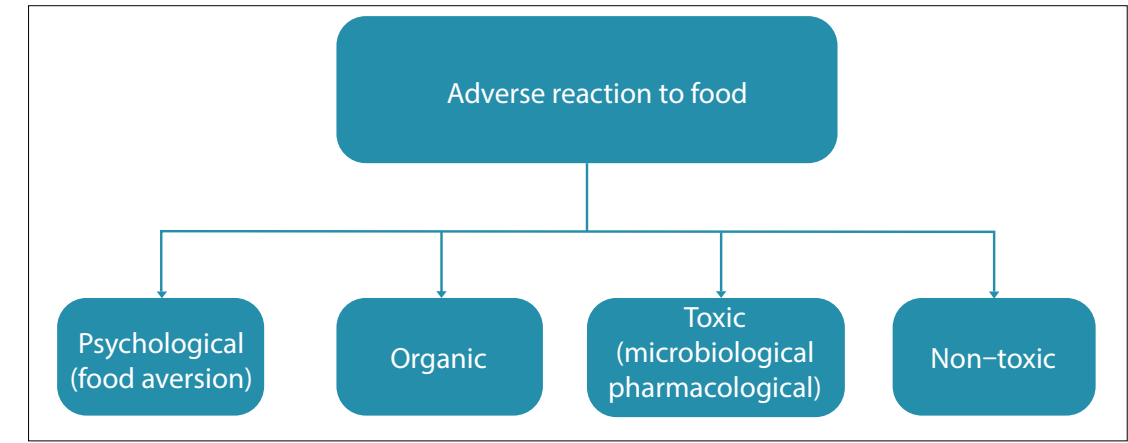

Fig 1. Adverse reactions to foods.

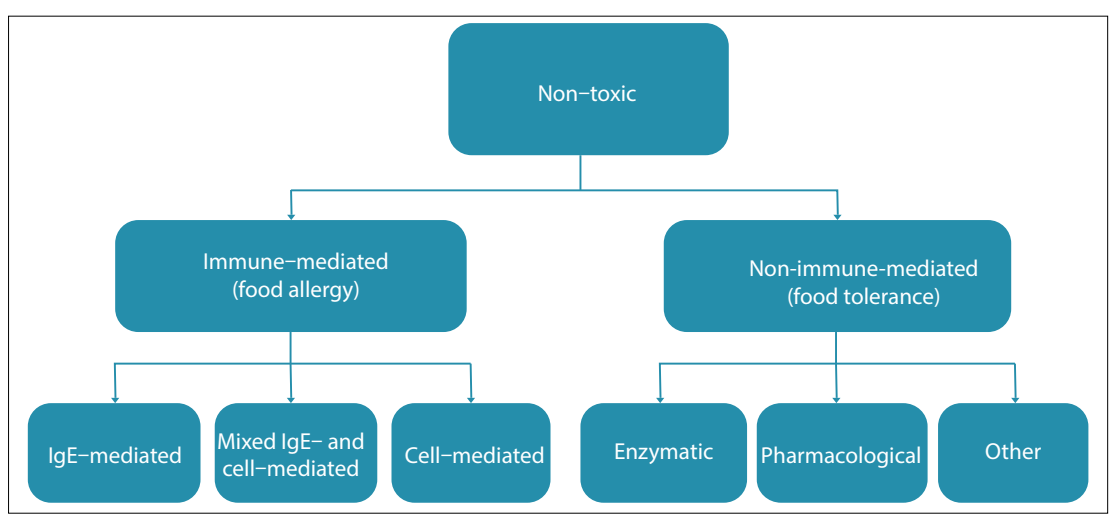

Fig. 2. Non-toxic food reactions.

Non-toxic reactions imply an individual hypersensitivity to the food, either immune- mediated (food allergy) or not immunemediated $^{[3]}$ (food intolerance) (Fig. 2). Non- 
toxic reactions only occur in susceptible individuals, rather than being experienced by all people exposed to that particular food. New nomenclature ${ }^{[4]}$ refers to 'food intolerance' as 'non-allergic food hypersensitivity'. Examples include lactose intolerance and hypersensitivity to alcohol or caffeine. This document focuses on immune-mediated reactions (food allergy) only.

Immunoglobulin E (IgE)-mediated food allergy is the clinical result of a type I immediate hypersensitivity reaction due to the presence of IgE antibodies to a specific food. Manifestations occur within minutes up to 2 hours after ingestion of the food. Symptoms usually recur on exposure to the food on every occasion. Symptoms may be mild or severe, associated with anaphylaxis. Symptoms may include urticaria, angio-oedema and other rashes, oral itching or swelling, and abdominal, airway, cardiovascular and central nervous system symptoms. IgE-mediated reactions may be divided into immediate-onset reactions (immediate in time) and immediate plus late-phase (in which the immediate-onset symptoms are followed by prolonged or ongoing symptoms).

Non-IgE-mediated food allergy. Non-IgE-mediated conditions include combined IgE- and cell-mediated conditions, such as atopic dermatitis and eosinophilic oesophagitis, and pure T-cell-mediated conditions, such as food protein-induced enterocolitis syndrome, allergic proctocolitis and enteropathy syndromes. ${ }^{[5]}$ Infants and young children are affected predominantly, and present with abdominal complaints such as pain, vomiting, diarrhoea, blood in the stools and failure to thrive. ${ }^{[6]}$ Diagnosing mixed or non-IgEmediated allergy is challenging. A clear cause-effect relationship between exposure to the suspected food and symptoms is not always possible, as symptoms develop over time and are more chronic in nature. Skin-prick tests (SPTs) and specific IgE tests to the allergen are usually negative. An elimination diet may be necessary to diagnose non-IgE-mediated type food allergy ${ }^{[7]}$ The suspected allergen should be excluded from the diet for $2-6$ weeks under dietetic guidance to assess for improvement of symptoms. After symptom improvement, a rechallenge is necessary to definitively prove causal relation..$^{[7]}$

\section{Multiple allergens or forms of allergies in individuals}

An individual may be allergic to multiple allergens, and each allergen may have a different mechanism of reaction and manifest differently, and therefore may require different modalities of diagnosis and management.

\section{Prevalence of IgE-mediated food allergy $^{[8]}$}

Despite the large number of foods that can cause IgE-mediated reactions, most prevalence studies have focused on the most common allergenic foods, namely cow's milk, hen's egg, peanut, tree nut, wheat, soya, fish and shellfish. ${ }^{[9]}$

Food allergy peaks in the first 2 years of life, and then diminishes towards late childhood as tolerance to several foods develops over time. Based on meta-analyses and large population-based studies, the true prevalence of food allergy varies from $1 \%$ to over $10 \%$, depending on the geographical area and age of patient being studied.

The prevalence of food allergy in SA is currently under investigation. The prevalence of IgE-mediated food allergy in SA children with moderate to severe atopic dermatitis is $40 \% ;{ }^{[10]}$ however, this represents a high-risk population for food allergy. An unselected population of 211 Xhosa high-school students showed an overall sensitisation to foods of 5\%. ${ }^{[1,12]}$ Preliminary data from the South African Food Sensitisation and Food Allergy (SAFFA) study, ${ }^{[13]}$ which is investigating food allergy in an unselected cohort of 1 - 3-year-olds, show a prevalence of SPT $\geq 1 \mathrm{~mm}$ to any food of $11.6 \%$, SPT $\geq 3 \mathrm{~mm}$ of $9.9 \%$ and SPT $\geq 7 \mathrm{~mm}$ of $4.2 \%$. Challenge-proven food allergy prevalence is $1.8 \%$, egg allergy $1.4 \%$ and peanut allergy $1.1 \%$.

\section{Food-related anaphylaxis and fatalities}

Food allergy appears to be the most common trigger of anaphylaxis in the community. One-third to half of anaphylactic episodes can be attributed to foods; ${ }^{[14]}$ this proportion seems to be higher in children, in whom food is responsible for up to $85 \%$ of anaphylactic reactions. ${ }^{[15]}$ In adults, shellfish and nut are the most common triggers of food-induced anaphylaxis, and in children, peanut, tree nut, milk and egg.

\section{Diagnosis of food allergy ${ }^{[16]}$}

No single test can be fully depended on in the diagnosis of food allergy. A detailed history is an essential initial step when a food allergy is suspected. ${ }^{[17]}$ History aids in identifying potentially involved food, in differentiating between potential IgE- and non-IgE-mediated disease and assessing severity of reactions.

Elements of history should be gathered separately for each food under consideration, as a single patient may experience different types of reactions with different foods, each of which will require different diagnostic and management strategies. Elements of a full history, including an allergy-focused family history, ${ }^{[18]}$ clinical history ${ }^{[19,20]}$ and dietary history, can be found in the review in this edition. ${ }^{[16]}$

History alone is however not diagnostic, ${ }^{[20]}$ and additional measures of sensitisation ${ }^{[16]}$ or food challenges ${ }^{[7]}$ are often required.

In suspected IgE-mediated allergy, SPT and/or measurement of serum specific IgE antibodies (ImmunoCAP) to suspected foods are used to prove sensitisation. Sensitisation does not, however, confirm clinical food allergy as these tests indicate an immunological response to the specific allergen, but the diagnosis requires a clear correlation between the test result and clinical reaction (by positive history or food challenge). ${ }^{[21]}$ Blind testing without suspicion of a reaction to a specific allergen is not advocated, as findings are often misleading by indicating asymptomatic sensitisation alone ${ }^{[22]}$ Tests for sensitisation to foods should not be performed when history indicates that those foods are tolerated.

No validated tests exist to confirm non-IgE- or mixed IgEand non-IgE-mediated food allergies. Diagnosis of this group of allergies depends on elimination of the suspected food, clearance of symptoms, and recurrence of symptoms on re-introduction of the food. ${ }^{[7]}$ In certain cases endoscopy with biopsy is indicated to evaluate the response to dietary changes. ${ }^{[5]}$

\section{Diagnostic tests to identify sensitisation in IgE-mediated food allergy ${ }^{[16]}$ \\ Skin-prick test (SPT)}

SPT is a valid and useful tool to confirm IgE-mediated sensitisation to a specific allergen. ${ }^{[21]}$ The choice of allergens to be tested should be guided by the history, but should be limited to the lowest necessary number to avoid false-positive results. The mean wheal diameter correlates with the likelihood of clinical allergy, but must be interpreted in the light of the clinical history. In individuals with atopic dermatitis, $95 \%$ positive predictive values (cut-off values for the mean wheal diameter in the common food allergens where a clinical reaction can be predicted in $95 \%$ of cases) have been described for immediate reactions but may be population-specific.

\section{Allergen-specific serum IgE}

Serum IgE specific to an individual allergen can be determined by ImmunoCAP assays. Cut-off values for the $95 \%$ positive predictive values of a clinical reaction to common food allergens are described 
(see Van der Spuy et al. ${ }^{[16]}$ in this issue) but may be populationspecific. ${ }^{[22]}$

Purified recombinant allergen-specific IgE tests against individual major allergen components in food may improve the diagnosis of clinical allergy and differentiate true food allergy from cross-reactivity. Certain component allergens are correlated with persistence of food allergy (e.g. ovomucoid in egg allergy and casein in cow's milk protein allergy), severity of reactions (e.g. Ara h 2 in peanut allergy) and lack of tolerance of extensively heated products (e.g. ovomucoid and casein).

\section{Elimination diets and challenges in the diagnosis of food allergy $^{[7]}$ \\ Diagnostic elimination diets}

Elimination diets become an important diagnostic tool when there is no clear cause and effect between ingestion of food and symptoms. This is most common when the reaction to the food is not immediate. In this instance, diagnosis can usually be made by elimination of food for a set period (usually between 2 and 6 weeks), followed by planned and intentional re-introduction.

Food re-introduction after (short-term) elimination diets for mildto-moderate non-IgE-mediated conditions may be performed in a safe clinical environment or with a cautious re-introduction at home. Foods should be re-introduced one at a time as a single ingredient rather than a composite food with 3 - 4 days of daily consumption before re-introduction of further allergens.

Patients who have had an IgE-mediated immediate reaction to food, a previous severe non-IgE-mediated reaction or a long period of food exclusion should not have a home challenge, but be challenged with a formal incremental food challenge in a controlled setting. ${ }^{[23]}$

\section{Food challenges ${ }^{[7]}$}

An incremental oral food challenge (OFC) is a test that consists of a gradual feeding of the suspected food under close observation. ${ }^{[24-27]}$ An OFC is preceded by a period of dietary elimination. OFC testing is the gold standard to diagnose clinical food allergy or to demonstrate tolerance. Incremental food challenge testing for IgE-mediated food allergy should be done by trained practitioners in centres that have experience in performing the procedure in an appropriate setting. OFCs do not necessarily signify the severity of reactions in real-life situation since challenges are gradual and are stopped the moment a reaction occurs. ${ }^{[28]}$ OFCs performed after a period of elimination may result in acute and severe reactions, even though the food was previously associated only with chronic symptoms. ${ }^{[25,29,30]}$ Food challenges include open, single-blind and double-blinded food challenges. ${ }^{[11,32]}$

OFC must be performed in a setting where resuscitation equipment is available in the event of a severe anaphylactic reaction. It can be performed in an outpatient or inpatient setting depending on patient clinical history. In high-risk cases (previous severe reactions or severe asthma), one-to-one nursing and insertion of an intravenous cannula may be required.

Challenges are terminated when a reaction becomes apparent. ${ }^{[26]}$ Standardised and pre-set criteria are available on when to stop challenges. ${ }^{[33]}$ Sample food challenge schedules are available for common food allergens in South Africa. ${ }^{[2]}$ Patients who tolerate the full dose 'pass' the challenge and are then advised to eat a full portion of the food at least twice a week to maintain tolerance. Subjects who have reactions have 'failed' the challenge, and should avoid the food and have education and risk reduction strategies implemented where appropriate.

Following a challenge, patients should be observed for a minimum of 2 hours following a negative challenge and 4 hours following a positive one.

\section{Elimination diets and dietary interventions for the management of food allergies ${ }^{[34]}$}

The primary therapy for food allergy is strict avoidance of the offending food or foods.

Dietary restriction should be individually tailored to meet the nutritional needs of each patient by a registered dietician who is experienced in food allergies. Patients should be re-evaluated at regular intervals to see if they have developed tolerance. This varies depending on the individual foods and whether it is an IgE- or nonIgE-mediated food allergy. Generally younger children with milk, soya and egg allergy should be reviewed every $6-12$ months and older children every $1-2$ years. Tree nut, fish and shellfish allergy may be lifelong, but re-evaluation should be performed every 2 - 4 years to determine whether rechallenges are appropriate or exclusion needs to be continued. ${ }^{[20]}$

\section{Education}

Education is the mainstay of dietary avoidance advice. Children, parents and carers should be educated on common ingredients, reading food labels and how to avoid allergens safely. Children and parents should also be made aware of appropriate safe, cost-effective, freely available and nutritionally adequate substitutes for the avoided foods.

\section{Cow's milk substitutes}

Parents of infants and young children with cow's milk protein allergy (CMPA) should be counselled on the most appropriate formula or milk substitute to use, ${ }^{[18]}$ taking into consideration the type of reaction experienced, severity of reaction, age of the patient (need for formula as the sole or a significant part of their diet), religious and cultural views, and the cost and palatability of the food.

\section{Breastfeeding}

Mothers of infants with CMPA who are breastfeeding should be supported and encouraged to continue breastfeeding. ${ }^{[1,35]}$ In extremely rare circumstances, an elimination diet may need to be followed by the mother, under dietary guidance to ensure nutritional adequacy. ${ }^{[17]}$

Hydrolysed cow's milk protein and elemental formulas The classification of feeds as partially hydrolysed, extensively hydrolysed or elemental depends on the degree of hydrolysis and thus the size of the resulting proteins or amino acids.

Partially hydrolysed infant formulas are not hypoallergenic (tolerated by $90 \%$ of subjects with proven CMPA) and are therefore not recommended for the treatment of CMPA, but may have a role in prevention of eczema or CMPA in high-risk individuals. ${ }^{[36-38]}$

Some extensively hydrolysed and amino-acid formulas are truly hypoallergenic. The recommended feed of choice for the dietary management of mild or moderate CMPA in infants not breastfed is an extensively hydrolysed cow's milk formula. ${ }^{[17,40]}$

The recommended formula for the dietary management of nonbreastfed infants and children with known severe CMPA is an amino-acidbased formula, ${ }^{[17]}$ which is the only completely non-allergenic formula.

Because of the high costs, an amino-acid-based formula has targeted use and is specifically recommended for: ${ }^{[17]}$

- infants with CMPA with severe or life-threatening symptoms

- patients with on-going symptoms on an extensively hydrolysed formula

- infants with severe growth faltering

- in rare circumstances where a stringent exclusion diet in the breastfeeding mother has failed to resolve symptoms in the infant. 


\section{Soya protein-based infant formulas}

Soya-based formulas may be useful in:

- infants with IgE-mediated CMPA with proven tolerance to soya ${ }^{[40]}$

- some cases of mild-to-moderate non-IgE-mediated CMPA, ${ }^{[4]}$ bearing in mind the increased risk of co-reactivity between CMPA and soya allergy in non-IgE-mediated conditions. This should be done under close supervision.

\section{Other}

Other mammalian milks are not suitable for individuals with CMPA because of extensive cross-reactivity. Plant-based milks, including rice and oat milks, are not nutritionally adequate and are inappropriate as a sole infant formula. Probiotics show a moderate risk reduction for eczema in approximately half of all studies, especially those with both pre- and postnatal administration of probiotics; ${ }^{[42]}$ however, at present a role for probiotics in the prevention or treatment of food allergy is not established and they should not be routinely prescribed.

\section{Severe food allergy and anaphylaxis:}

Treatment, risk assessment and risk reduction ${ }^{[43]}$

\section{Anaphylaxis}

Anaphylaxis may be fatal if not recognised and managed appropriately with treatment given rapidly. The drug of choice for treatment of anaphylaxis is adrenaline administered by the intramuscular (IM) route (not subcutaneously) in the mid-anterolateral thigh with other drugs given as adjunctive therapy. ${ }^{[44,45]}$

The following steps should be instituted in the management of anaphylaxis:

- eliminate additional exposure to the allergen

- give IM injection(s) of adrenaline

- call for help

- place the patient in a recumbent position with lower limbs elevated and do not allow the patient to stand quickly

- give supplemental oxygen

- administer intravenous fluid if hypotensive or incomplete response to IM adrenaline

- administer nebulised bronchodilators for lower airway obstruction, and/or nebulised adrenaline for stridor

- medications above may be repeated if there is inadequate response to therapy

- administer adjunctive therapy (antihistamines and corticosteroids)

- admit subjects with anaphylaxis for close observation for biphasic reactions

- initiate or review risk-reduction strategies after recovery from the acute episode.

Risk factors for the severity of allergic reactions to foods Factors associated with increased risk in epidemiological studies ${ }^{[46]}$ include coexisting asthma (and current poor asthma control), previous severe reactions, delayed administration of adrenaline, adolescents and young adults, reaction to trace amounts of foods, use of non-selective $\beta$-blockers and those remote from medical care.

\section{Elements of risk reduction ${ }^{[43,46]}$}

These include:

- Education and provision of a written emergency treatment plan on allergen avoidance, early symptom recognition and appropriate emergency treatment.

- Risk assessment and stratification with provision of injectable adrenaline (preferably via auto-injector) if necessary. Adrenaline auto-injectors (e.g. EpiPen) are more convenient options than ampoules and syringes which may be difficult to use quickly or accurately, especially by parents/patients in emergency situations. ${ }^{[47]}$ EpiPen is available in a $0.15 \mathrm{mg}$ dose for subjects weighing $8-25 \mathrm{~kg}$ and a $0.3 \mathrm{mg}$ dose for subjects who weigh $>25 \mathrm{~kg}$.

- Training and provision of written instructions in the indications for and method of administration of adrenaline.

- Patients and caregivers should be educated about foods that should be avoided and available alternatives.

- Patients should be informed about reading labels. Clear guidelines should also be set for labelling foodstuffs. This includes products which should be clearly linked to the name of the allergen to avoid confusion.

- The diagnosis of food allergy should, with permission, be communicated to all relevant caregivers, including school teachers. A MedicAlert necklace or bracelet should be worn by the patient at all times.

- Patients should be encouraged to join an appropriate patient support organisation.

\section{Conclusion}

Subjects may have reactions to more than one food, and different types and severity of reactions to different foods may coexist in one individual. A detailed history directed at identifying the type and severity of possible reactions is essential for every food allergen under consideration. SPTs and specific IgE (ImmunoCAP) tests prove IgE sensitisation rather than clinical reactivity. The magnitude of sensitisation combined with the history may be sufficient to ascribe causality, but where this is not possible an incremental food challenge may be required to assess tolerance or clinical allergy. For milder non-IgE-mediated conditions, a diagnostic elimination diet may be followed with food re-introduction at home to assess causality.

The primary therapy for food allergy is strict avoidance of the offending food/s taking into account nutritional status and provision of alternative sources of nutrients. Acute management of severe reactions requires prompt IM administration of adrenaline $0.01 \mathrm{mg} / \mathrm{kg}$ and basic resuscitation. Adjunctive therapy includes antihistamines, bronchodilators and corticosteroids. Subjects with food allergy require risk assessment and those at increased risk for future severe reactions require the implementation of risk-reduction strategies including education of the patient, families and all caregivers (including teachers), the provision of a written emergency action plan, a MedicAlert necklace or bracelet and injectable adrenaline (preferably via auto-injector) where necessary. 